\title{
PAPER
}

\section{Blind source separation using recursive TDD: Introduction of a threshold on separation performance}

\author{
Xuebin $\mathrm{Hu}^{*}$ and Hidefumi Kobatake ${ }^{\dagger}$ \\ Graduate School of Bio-Applications and Systems Engineering, \\ Tokyo University of Agriculture and Technology, \\ 2-24-16, Naka-cho, Koganei, 184-8588 Japan
}

(Received 19 December 2001, Accepted for publication 30 September 2002)

\begin{abstract}
For most of the blind separation methods of convolutive mixtures, the parameters of unmixing filter are derived in frequency domain. This leads to a seldom mentioned but important problem that generally the independence assumption between source signals collapses in frequency domain because of the inadequate samples. There exists correlation at each frequency bin. Sometimes it is too high to be neglected and consequently degrades the performance of all the BSS methods in various degrees. In this paper, we propose a recursive algorithm for lowering the unfavorable effect from the correlation, and combine it with the TDD-based blind separation method proposed by S. Ikeda and N. Murata. The bin mixtures are separated into the components of the sources as practical instead of the independent bins as achieved by the conventional method. The signal-to-noise ratio is greatly increased at certain bins, which results in a much better separation.
\end{abstract}

Keywords: BSS, TDD, Frequency domain, Recursive method

PACS number: 43.60.Gk

\section{INTRODUCTION}

Blind source separation (BSS) has received extensive attention in signal and speech processing, machine intelligence, and neuroscience communities. The goal of BSS is to recover the unobserved original sources without any prior information given only the sensor observations that are unknown linear mixtures of the independent source signals. A variety of successful BSS methods have been developed including [2-12].

In real environment acoustic sources separation, due to the multi-path effect and reverberation, the signals impinged on an array of microphones are convolutive mixtures of sources. BSS may be implemented in time domain by learning the time-domain coefficients of the unmixing filter. However, the filters may need to be thousands of taps long to properly invert the mixing. Computationally it may be lighter to move to the frequency domain as convolutions with long filters in the time domain becomes efficient multiplications in the frequency domain under certain conditions [1,13]. This has the great advantage that we can use any desired method. A number

\footnotetext{
*e-mail: huxb@cc.tuat.ac.jp

†e-mail: kobatake@cc.tuat.ac.jp
}

of methods for convolutive source separation have been proposed [1,14-18]. S. Ikeda and N. Murata applied the time delayed decorrelation method (TDD) proposed by L. Molgedey and H. G. Schuster [3] and proposed a frequency domain convolutive mixture separation method taking advantage of the temporal structure of speech signal [1].

The commonly mentioned disadvantage in frequency domain implementation is that the standard ICA indeterminacy of scaling and permutation appears at each output frequency bin. Various methods using different continuity criteria as listed in [13] have been reported.

There is another disadvantage, which is not mentioned yet but is thought to be very important. It is the independence assumption between source signals collapsed in frequency domain. This is because the BSS is normally implemented on a short time period of observations due to the dynamic mixing process in real environment. Frequency domain implementation leads to much less samples than in time domain. Consequently, the estimated correlation function often includes large estimation error and can no longer be expected to be zeros. We say that the frequency representations of the source signals corresponding to the observations of limited samples are correlated. The existing correlation obviously disobeys the commonly adopted basic assumption of ICA that the sources should 
be independent with each other. It decreases the performance of ICA in different degree, sometimes very seriously, at various frequency bins whatever the separation method is used. Furthermore, it degrades the performance of the solution to the ambiguity of scaling and permutation problem.

This paper proposes a recursive algorithm for the correlation problem occurred in frequency domain implementation. A criterion on the performance of separation is introduced and with which we distinguish the improper separation and correct it using a trial-and-error scheme. In this paper, the recursive algorithm is combined with the temporal structure based TDD method proposed by S. Ikeda and N. Murata. Section 2 describes the mixture model, and the correlation problem in frequency domain implementation. Section 3 briefs the BSS system. Section 4 gives the definition and explanation on the recursive algorithm. At last, we present the simulation results, and conclude the paper.

\section{THE PROBLEM}

\subsection{Mixture Model in Frequency Domain}

First we give a formulation of the problem. Source signals are assumed to be independent with each other, zero mean, and are denoted by a vector $s(t)=\left(s_{1}(t), \cdots, s_{N}(t)\right)^{\mathrm{T}}$. When the signals are recorded in a real environment, the observations can be approximated with convolutive mixtures of source signals,

$$
\boldsymbol{x}(t)=\boldsymbol{A} * \boldsymbol{s}(t)=\left(\sum_{i} a_{i k} * s_{i}(t)\right),
$$

where $\boldsymbol{A}$ is an unknown polynomial matrix, $a_{i k}$ is the impulse response from source $i$ to microphone $k$, and the symbol $*$ refers to convolution. In frequency domain, the convolutive mixing problem is decomposed into multiple instantaneous mixing problems.

$$
X(f, t)=A(f) S(f, t) .
$$

The instantaneous mixing problem then can be solved using any desired method. With the derived unmixing filter $\boldsymbol{W}(f)$, we recover the source signals by

$$
\hat{\boldsymbol{S}}(f, t)=\boldsymbol{W}(f) \boldsymbol{X}(f, t) .
$$

\subsection{Independence Collapses at Frequency Bin}

Due to the limited number of samples, frequency components of source signals are no longer independent with each other. There exist correlations in certain degree at various frequency bins [19].

For evaluation of the correlation between $s_{1}(f, t), \cdots$, $\boldsymbol{s}_{K}(f, t)$, we define that,

$$
\boldsymbol{V}(f)=\operatorname{diag}\left(\left\langle\boldsymbol{S}(f, t) \boldsymbol{S}^{\mathrm{H}}(f, t)\right\rangle\right)-\left\langle\boldsymbol{S}(f, t) \boldsymbol{S}^{\mathrm{H}}(f, t)\right\rangle,
$$

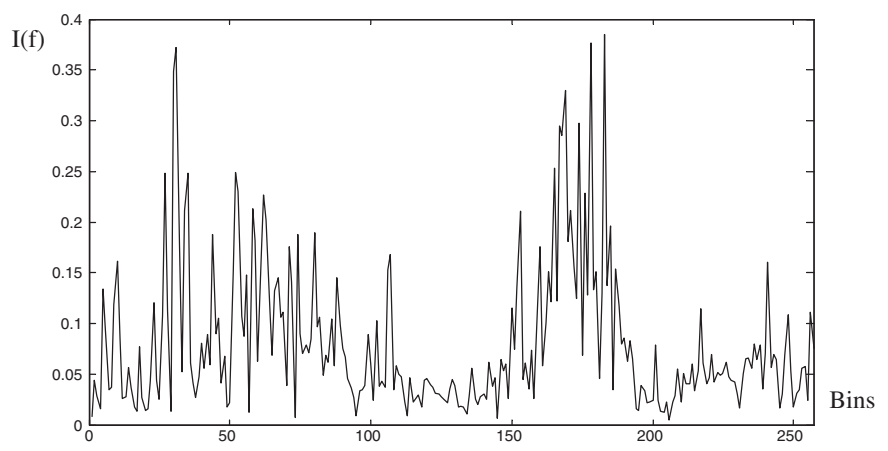

Fig. 1 Distribution of correlation against frequency.

where $\langle\cdot\rangle$ denotes the expectation operator, and $\left\langle\boldsymbol{S}(f, t) \boldsymbol{S}^{\mathrm{H}}(f, t)\right\rangle$ is the normalized covariance matrix. The independence between the frequency components of source signals, denoted as $I(f)$, is quantified by the Frobenius norm of $\boldsymbol{V}(f)$. It is further normalized and defined as,

$$
\boldsymbol{I}(f)=\|\boldsymbol{V}(f)\|=\sqrt{\sum_{l \neq k}\left|\boldsymbol{V}_{i k}(f)\right|^{2} /\left(N^{2}-N\right)}
$$

The higher the value $\boldsymbol{I}(f)$ is, the lower the independence will be. Figure 1 shows the independence distribution against frequency between the two speeches, "Good morning" and "Konbanwa," which are downloaded from S. Ikeda's homepage. The signals are about one second in length, and the sampling rate is $16 \mathrm{kHz}$. The DFT length is 512 samples $(32 \mathrm{~ms})$ and the window shift is 5 samples. Hamming window is used. It is noted that the correlation may vary in some degree when using different frame length and window shift [19]. But it will not cause significant change to it.

Regardless of the separation method, the off-diagonal elements of the covariance matrix of the separated frequency components are to be minimized as practical, ideally minimized to zeros. In other words, ICA will make $I(f)$ close to or equal to zero. The existing correlations between the sources as shown in Fig. 1 apparently show that ICA will not work perfectly in such condition. As such, measure for eliminating the unfavorable effect from the correlation is necessary.

\section{BSS SYSTEM}

The BSS system proposed in this paper is summarized as follows. First, the observed mixtures or a part of them are decomposed into frequency representations by performing discrete Fourier Transformation. The mixed frequency bins are then separated into the frequency components of the original sources using the proposed recursive TDD algorithm (rTDD). Executing solution to the ambiguity of scaling and permutation, the bin unmixing filters are then inverted into time domain unmixing filter. Figure 2 shows the flow chart of the BSS system. 


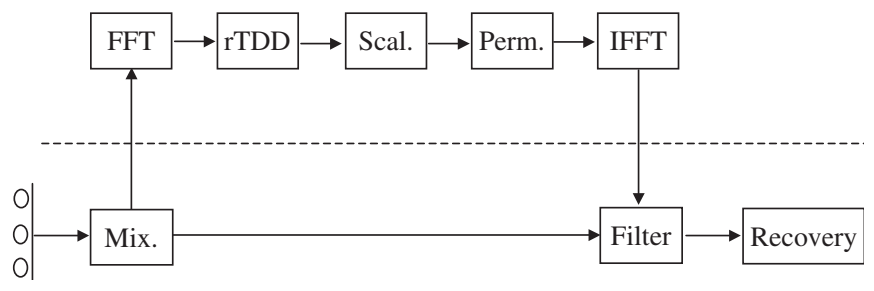

Fig. 2 Flow chart of BSS system. Mix.: Mixture, Scal.: Scaling, Perm.: Permutation. The part above the dot line is in frequency domain, and the part below is in time domain.

\subsection{Bin Mixture Separation}

As the frequency representations of the observations are the instantaneous mixtures of the frequency components of sources, a variety of BSS methods could be used for this purpose. However, due to the correlation problem mentioned in section 2, the common assumption of ICA does not strictly hold. Pointing to it, we propose a recursive algorithm and combine it with the TDD based approach. It is used to derive the unmixing filter $\boldsymbol{W}(f)$ in Eq. (3). The bin mixtures are then separated into the frequency components of the original sources at the most, instead of the "independent bins" as achieved by the conventional methods. It is to be described in detail in section 4 .

\subsection{Scaling and Permutation [1]}

Normally, ambiguity of scaling and permutation is acceptable for ICA. However, it will cause serious problem in frequency domain. We use the commonly adopted solution to ambiguity of scaling; i.e., by putting back the separated frequency components to the sensor with the inverse matrices to solve the problem of irregular amplitude. The unmixing filter after solving the ambiguity of scaling, $\boldsymbol{W}_{i, f^{\prime}}$ is defined as

$$
\boldsymbol{W}_{i, \mathrm{f}}{ }^{\prime}=\boldsymbol{W}_{\mathrm{f}}^{-1} \delta(i, i) \boldsymbol{W}_{\mathrm{f}},
$$

where $\boldsymbol{W}_{\mathrm{f}}$ denotes the derived bin unmixing filter by rTDD, $\boldsymbol{W}_{i, \mathrm{f}}{ }^{\prime}$ denotes the unmixing filter that outputs the $i$-th source signal, and $\delta(i, i)$ denotes a "delta matrix" of which only the $(i, i)$ element equals to one and all the remaining elements are zeros.

Concerning permutation, Ikeda et al. proposed a method based on the observation that if the original source is the same, the adjacent envelopes are similar. It is used in our system. The operator to take the envelope, $\varepsilon$ is defined as

$$
\varepsilon \hat{\mathbf{s}}_{\mathrm{f}}\left(t_{\mathrm{s}} ; i\right)=\frac{1}{2 M} \sum_{t_{\mathrm{s}}^{\prime}=t_{\mathrm{s}}-M}^{t_{\mathrm{s}}+M} \sum_{k=1}^{n}\left|\hat{\boldsymbol{s}}_{k, \mathrm{f}}\left(t_{\mathrm{s}}{ }^{\prime} ; i\right)\right|,
$$

where $\hat{\boldsymbol{s}}_{\mathrm{f}}\left(t_{\mathrm{s}} ; i\right)$ denotes the output of the $i$-th unmixing filter $\boldsymbol{W}_{i, \mathrm{f}}{ }^{\prime}$, i.e. the frequency component of the $i$-th source, and $\hat{\boldsymbol{s}}_{k, \mathrm{f}}\left(t_{\mathrm{s}}{ }^{\prime} ; i\right)$ denotes the input of the $i$-th source component into the $k$-th $(k=1, \cdots, n)$ sensor. Here, $M$ is the number of time steps for taking the moving average. Criteria for similarity among the envelopes of the same frequency is defined in [1] as

$$
\operatorname{sim}(f)=\sum_{i \neq k} \frac{\varepsilon \hat{\boldsymbol{s}}_{\mathrm{f}}\left(t_{\mathrm{s}} ; i\right) \cdot \varepsilon \hat{\boldsymbol{s}}_{\mathrm{f}}\left(t_{\mathrm{s}} ; k\right)}{\left\|\varepsilon \hat{\mathbf{s}}_{\mathrm{f}}\left(t_{\mathrm{s}} ; i\right)\right\|\left\|\varepsilon \hat{\boldsymbol{s}}_{\mathrm{f}}\left(t_{\mathrm{s}} ; k\right)\right\|},
$$

where "." denotes inner product, and \|\| denotes the norm. Denoting the permutation solution as $\sigma_{\mathrm{f}}(i)$, the unmixing filter then is derived as

$$
\boldsymbol{W}_{i, \mathrm{f}^{\prime \prime}}=\boldsymbol{W}_{\mathrm{f}}^{-1} \delta\left(\sigma_{\mathrm{f}}(i), \sigma_{\mathrm{f}}(i)\right) \boldsymbol{W}_{\mathrm{f}} .
$$

\subsection{Time Domain Filter}

By performing inverse DFT and multiplying the hamming window on the separated frequency bins or on $\boldsymbol{W}_{i, \mathrm{f}}{ }^{\prime \prime}$, we can directly recover the source signals or derive the unmixing filter in time domain respectively. The time domain unmixing filter is derived as follows.

$$
\boldsymbol{W}_{i, \mathrm{t}}=F^{-1}\left[\boldsymbol{W}_{i, \mathrm{f}^{\prime \prime}}\left(f_{k}\right) H\left(f_{k}\right)\right] \cdot \operatorname{ham}(t)
$$

where $F^{-1}$ denotes inverse Fourier transformation, $\operatorname{ham}(t)$ denotes the Hamming window, and $H\left(f_{k}\right)$ is a circular time shift operator. It is reported in [16] that when the FFT length is $N$, a time shift of about $N / 2$ is experimentally good, i.e., $H\left(f_{k}\right)=e^{j \pi k}$.

\section{RECURSIVE TDD}

The collapse of independence in frequency domain implementation usually results in inadequate separation in the related frequency bin for any of the BSS methods. The proposed method using recursively applied TDD tends to eliminate the unfavorable effect caused by the correlation.

\subsection{Criterion}

What we had to do first is to find a criterion for making the decision whether a bin mixture separation is acceptable or not. Because normally we do not have the real source signals, we can not directly distinguish whether a bin of frequency components of original sources are relatively correlated or not, and subsequently whether the separation of their mixtures is acceptable or not.

Fortunately, we can make use of the separated frequency components achieved by conventional ICA. ICA assumes $S(f, t)$ is independent and separates the frequency mixtures into mutually independent components. However $\boldsymbol{S}(f, t)$ is not perfectly independent due to the estimation error resulting from the limited samples. Sometimes the estimation error is too large to be neglected and make the separation completely failed. In such case, the separated components are still mixed with each other. An illustrative example is given in Fig. 3(a). Y1 and Y2 are the separated results achieved by conventional TDD, and S1, 

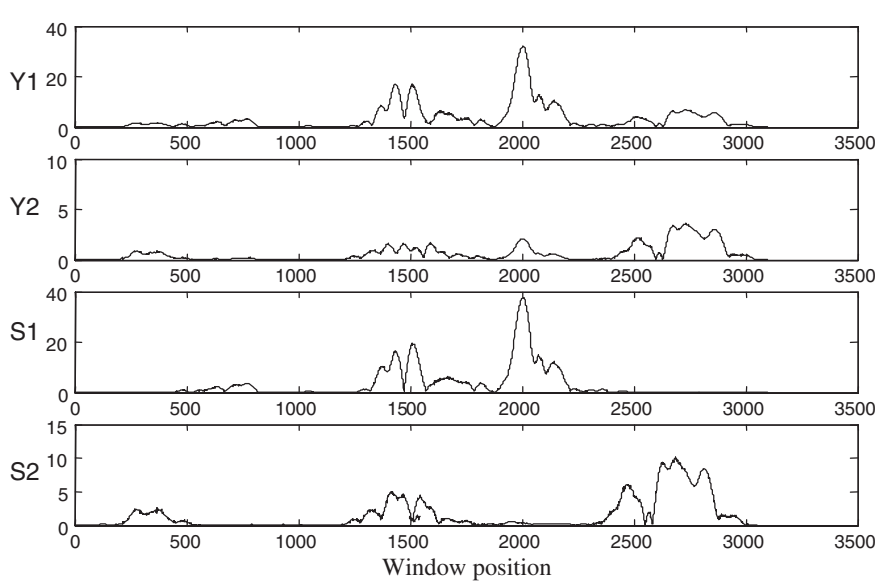

(a)

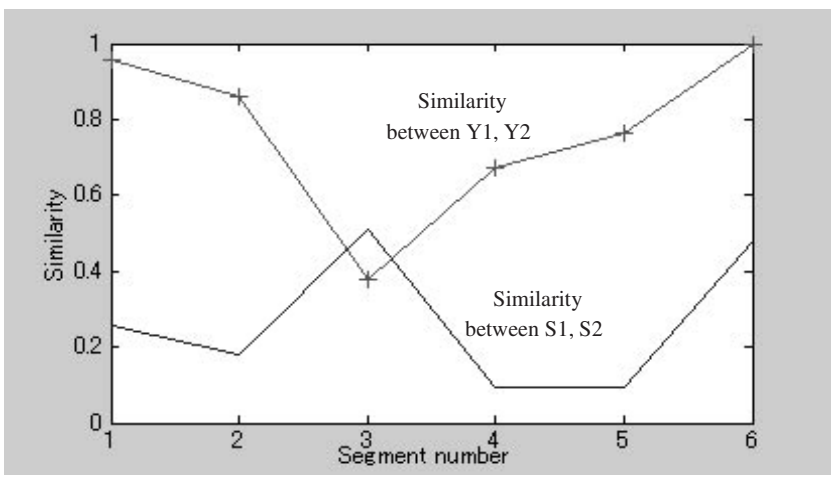

(b)

Fig. 3 (a) An example of improper separation by conventional TDD. (b) Segmental similarities between improperly separated signals and original signals.

S2 are the components of original sources. The waveforms are the norms of the complex-valued signals respectively.

Although Y1 and Y2 are uncorrelated with each other, comparing with $\mathrm{S} 1$ and $\mathrm{S} 2$, it is obvious from Fig. 3 that the waveforms of $\mathrm{Y} 1$ and $\mathrm{Y} 2$ are quite similar at certain segment pairs, for example at $[1,900,2,200]$ and $[2,400$, $3,000]$. This is because $\mathrm{Y} 1$ and $\mathrm{Y} 2$ have not been properly separated and still mixed with each other.

We divide the separated frequency components, which are achieved by conventional separation method, into continuous segments, and adopt the averaged segmental similarity as criterion to make the judgment. Divide the separated components $\hat{\boldsymbol{S}}(f, t)$, into a series of $\hat{\boldsymbol{S}}(f, l)$,

$$
\hat{\boldsymbol{S}}(f, l)=\hat{\boldsymbol{S}}(f,(l-1) \times m+1: l \times m),
$$

where, $l$ is the segment sequence number and $m$ is the segmental length. The averaged segmental similarity (ASS) is defined as

$$
A S S_{\mathrm{f}}=\frac{1}{L} \sum_{l=1}^{L} \frac{\left\|\hat{\boldsymbol{S}}_{1}(f, l) \cdot \hat{\boldsymbol{S}}_{2}(f, l)^{\mathrm{H}}\right\|}{\left\|\hat{\boldsymbol{S}}_{1}(f, l)\right\|\left\|\hat{\boldsymbol{S}}_{2}(f, l)\right\|} .
$$

Figure 3(b) shows the segmental similarities between Y1, Y2 and S1, S2, respectively. They are divided into six segment pairs with $m$ equals to 500, respectively. We can see that the average of the segmental similarities of improper separation is higher than that of complete separation.

It is observed that those with higher averaged segmental similarity tend to be improperly separated, and need to be further handled. Experimentally, it is found that when it is greater than $0.5-0.6$, the separation tends to be improper. And if it is less than $0.4-0.5$, generally it is acceptable.

\subsection{Recursive Method}

Next, we intend to correct the judged improper bin separation. The ideal procedure is to make the separation work on the mixtures of which the original source signals are less correlated. However, the existing correlation comes from the whole signal components, it is impossible to correct an improper separation from its causality.

We use a trial-and-error scheme to find the segments with bad effect on the separation. Because the number of combination of segment which may have bad effects on TDD becomes huge, it is not practical to search every combination. In consideration that the segment pair with higher variance (power) has higher weight in the learning of the unmixing filter, we select to remove the segment pair with high variance. Experiments show that if we take away the segment pair with high variance, and use the remaining mixtures to perform ICA again, it tends to provide a better derivation of the unmixing filter. And filtering the original mixtures by the derived filter, better separation could be expected. Figure 4 gives the illustration of this method.

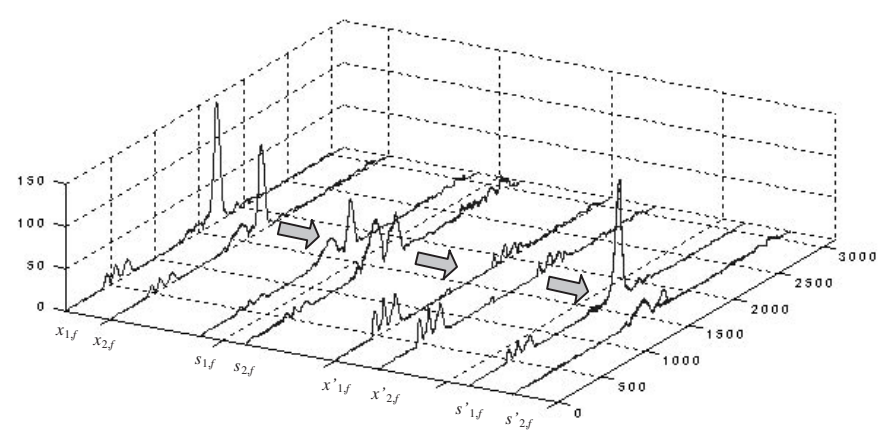

Fig. 4 Illustration of recursive TDD method. Waveforms $x_{1, \mathrm{f}}$ and $x_{2, \mathrm{f}}$ are the frequency mixtures. $s_{1, \mathrm{f}}$ and $s_{2, \mathrm{f}}$ are the separated frequency components achieved by conventional TDD. Separation at this bin is improperly implemented. Waveforms $x_{1, \mathrm{f}^{\prime}}$ and $x_{2, \mathrm{f}^{\prime}}$ are the remaining mixtures. Two segment pairs around sample No. 1500 have been removed. The amplitudes of $x_{1, \mathrm{f}^{\prime}}$ and $x_{2, \mathrm{f}^{\prime}}$ were amplified by two times for easy view. $s_{1, \mathrm{f}^{\prime}}$ and $s_{2, \mathrm{f}^{\prime}}$ are the result achieved by the recursive TDD on the remaining mixtures. Here, the waveform refers to the norm of complex-valued signals. 


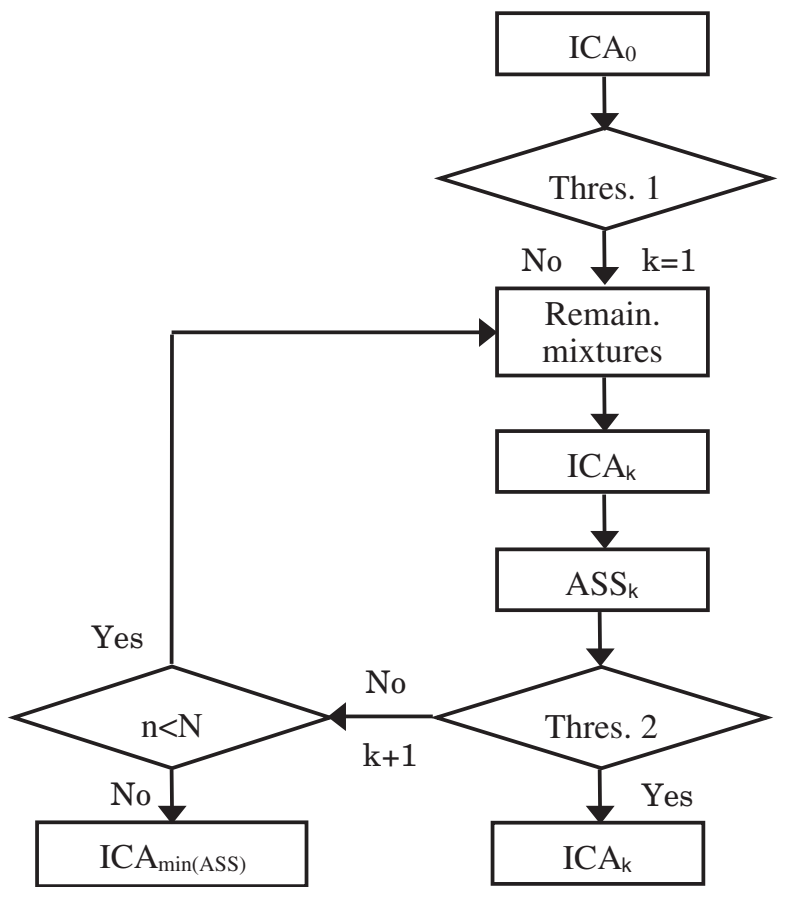

Fig. 5 Process flow of rTDD. Thres. 1 judges the separation acceptable or not. Thres. 2 judges the loop should be stopped or not. $N$ is the maximal iteration times. When iteration times is greater than $N$, stop the loop and select the separation that outputs the minimum of ASS as final result.

It often happens that after we take away the segment with highest variance, it still does not provide better separation, and on the contrary, the value of ASS increases. The unmixing filter becomes far from the ideal one. Then we need to take away more segments with higher variance in the remaining mixture.

In addition to the threshold designated for the judgment of improper separation, a lower threshold of acceptance is also designated. It is used to make the decision whether to accept the new separation and stop the loop or not. The process flow of the recursive ICA is depicted in Fig. 5. First if the criterion output is greater than the designated threshold, the separation is considered to be improper and needed to be corrected. We remove the segments with high variance in sequence until it is below the acceptance threshold. $N$ is the designated maximal iteration times. When the maximal iteration times is reached and the acceptance threshold still cannot be satisfied, the separation that outputs the minimum ASS is selected as a final result. It is noted that we should not remove too many segments as too short samples cannot ensure good derivation of the unmixing matrix.

\subsection{Time Delayed Decorrelation Method}

In this paper, we combine the time delayed decorrelation algorithm (TDD) with our method. There are several time delayed decorrelation algorithms proposed using PCA technique [2-4], and the method proposed by L. Molgedey and H. G. Schuster [3] has been extended into frequency domain for solving the convolutive mixture problem [1]. The main advantage of TDD algorithm is the computational efficiency in computing the cross-filters since it only uses straightforward calculations and no adaptation is necessary.

The time delayed decorrelation method consists of two steps, sphering and rotation. In time domain, the sphering operation (PCA filter) is defined below:

$$
\boldsymbol{x}^{\prime}(t)=\boldsymbol{U} \boldsymbol{x}(t), \quad \boldsymbol{U}=\sqrt{\Lambda^{-1}} \Gamma^{\mathrm{T}},
$$

where, $\Gamma$ and $\Lambda$ are a unitary orthogonal matrix and a diagonal matrix, which satisfy $\left\langle\boldsymbol{x}(t) \boldsymbol{x}(t)^{\mathrm{T}}\right\rangle=\Gamma \Lambda \Gamma^{\mathrm{T}}$.

In the second step, $\boldsymbol{x}^{\prime}(t)$, the output of PCA filter, is further handled using rotation filter $\boldsymbol{C}$.

$$
\boldsymbol{s}(t)=\boldsymbol{C} \boldsymbol{x}^{\prime}(t) .
$$

Rotation filter $\boldsymbol{C}$ is determined by removing the offdiagonal elements of the correlation matrix at several time delays. The implementation is to find an orthogonal matrix $C$ that minimizes

$$
\sum_{k=1}^{r} \sum_{i \neq j}\left|\left(\boldsymbol{C} \boldsymbol{M}_{k} \boldsymbol{C}^{\mathrm{T}}\right)_{i j}\right|^{2}
$$

where $\left(\boldsymbol{C} \boldsymbol{M}_{k} \boldsymbol{C}^{\mathrm{T}}\right)_{i j}$ denotes the $i j$-element of matrix $\boldsymbol{C} \boldsymbol{M}_{k} \boldsymbol{C}^{\mathrm{T}}, k$ denotes the index corresponding to the time delay $\tau_{k}$ and

$$
\boldsymbol{M}_{k}=\left\langle\boldsymbol{x}^{\prime}(t) \boldsymbol{x}^{\prime}\left(t+\tau_{k}\right)^{\mathrm{T}}\right\rangle, \quad k=1, \cdots, r
$$

The unmixing matrix is then expressed as

$$
\boldsymbol{B}=\boldsymbol{C U}
$$

\section{EXPERIMENTAL RESULTS}

\subsection{Effect of rTDD}

First, the performance of the recursive algorithm was demonstrated in the simplest condition (test 1). The same signals as in section 2.2 were used, where the mixing matrix was $[1,0.5 ; 0.7,1]$. The mixtures were divided into frames of $32 \mathrm{~ms}$ length each and the window shift $\Delta T$ was 5 samples. The length of FFT is 512, and Hamming window was used. The $r$ in Eqs. (15) and (16) was set to 40. The designated threshold and the acceptance threshold were set to 0.55 , and 0.4 respectively. Parameter $l$ or $m$ in Eq. (11), is the one needed to be decided in the recursive algorithm. Generally, the shorter the $m$ is, or the bigger the $l$ is, the better the SNR will be. However, too big value of the $l$ will lead to heavy computation quantity. Experimentally, segment number from 15 to 20 is adequate to provide a considerable improvement. Here $l$ and $m$ were set to 15 and 200, respectively. The maximal iteration times $N$ was 7. We compared our result with that of the previous 
method.

Since we know the true sources and the mixing rates, the signal-to-noise ratio (SNR) was used to evaluate the performance at each bin, and is defined as follows:

$$
S N R_{i j}=10 \log _{10} \frac{\sum_{t} \operatorname{signal}_{i}(t ; j)^{2}}{\sum_{t} \operatorname{error}_{i}(t ; j)^{2}}
$$

where

$$
\begin{aligned}
& \operatorname{signal}_{i}(t ; j)=a_{i j} s_{j}(t) \\
& \operatorname{error}_{i}(t ; j)=\hat{s}_{i}(t ; j)-\operatorname{signal}_{i}(t ; j)
\end{aligned}
$$

Figure 6 shows a significantly improved example achieved at 105 th frequency bin. The 1st and 4th waveforms are the norm of the separation results $\hat{\boldsymbol{S}}(f, t)$ achieved by using the temporal structure based TDD method. Comparing with the 3rd and 6th waveforms of the original sources, the 1st and 4th waveforms are far away from the 3 rd and 6 th waveforms respectively. It shows that separation at this bin is improperly implemented. The 2 nd and 5th waveforms are those of the result of the recursive algorithm combined with the same ICA method. They are very close to the original sources and obviously much better than those obtained by the conventional method.

It is emphasized that the recursive algorithm also contributes to the solution for the scaling and permutation problem. Apparently the result shown in Fig. 6 is better both in amplitude and waveform, which makes it easy to make a proper selection on the ambiguity of permutation.

Figure 7 shows the comparison of SNR and ASS between the recursive algorithm and the temporal structure based method. Considerable improvement in SNR has been achieved by our algorithm. At the designated threshold, about 40 percent of the bins were judged improper, and were further handled by the recursive algorithm. As a

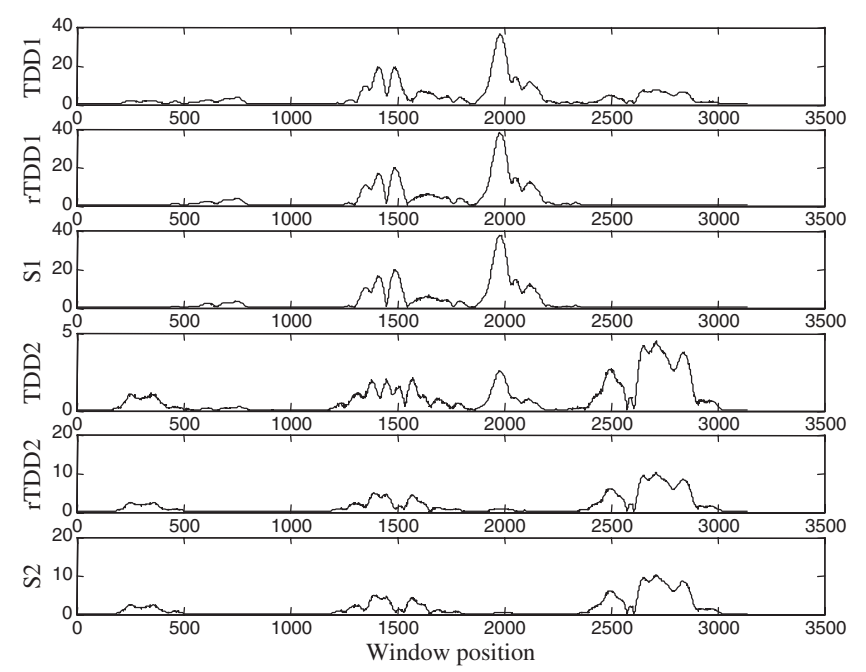

Fig. 6 An example of significant improvement at the 105th frequency bin.
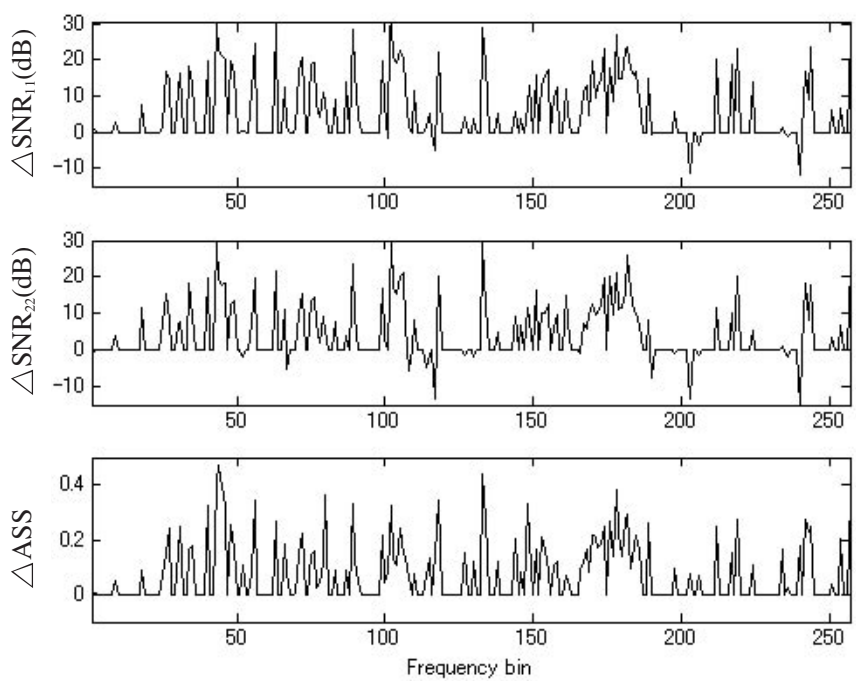

Fig. 7 The upper two figures show the improvement of SNR achieved by the recursive method compared to the conventional TDD at source 1 and 2, respectively. $\Delta S N R=S N R(\mathrm{rTDD})-S N R(\mathrm{TDD})$. The lower figure depicts the variation of ASS before and after the recursive method.

Table 1 SNR $(\mathrm{dB})$ of test 1.

\begin{tabular}{rcccc}
\hline & $\mathrm{SNR}_{11}$ & $\mathrm{SNR}_{12}$ & $\mathrm{SNR}_{21}$ & $\mathrm{SNR}_{22}$ \\
\hline TDD & 16.82 & 14.22 & 10.88 & 17.34 \\
rTDD & 23.64 & 19.68 & 17.61 & 22.67 \\
\hline
\end{tabular}

result, they give better SNR's. Accompanying with the improvement of SNR, ASS decreased about 0.17 in average. It is noted that at a few bins, SNR became worse. However, with the recursive algorithm, an overall improvement is achieved. Table 1 gives the overall signal-tonoise ratios achieved by TDD and our method.

\subsection{Convolutive Mixture}

We tested the method on convolutive mixtures. We trialed on 24 pairs of speech signals from ASJ Continuous Speech Database. They were mixed using the filters in Eq. (21).

$$
\begin{aligned}
& A_{11}=0.9+0.5 z^{-1}+0.3 z^{-2} \\
& A_{12}=-0.7 z^{-5}-0.3 z^{-6}-0.2 z^{-7} \\
& A_{21}=0.5 z^{-5}+0.3 z^{-6}+0.2 z^{-7} \\
& A_{22}=0.8-0.1 z^{-1}
\end{aligned}
$$

Same parameters were used as in section 5.1 with the exception that: window shift was $20, l$ and $m$ were 20 and 77 , and $N$ was 10 . Two seconds long of mixed speech signals were used to learn the unmixing filter. The averaged noise reduction ratios (NRR) are shown in Fig. 8. The recursive approach gave about $3.4 \mathrm{~dB}$ higher NRR in average than that of the conventional TDD. 


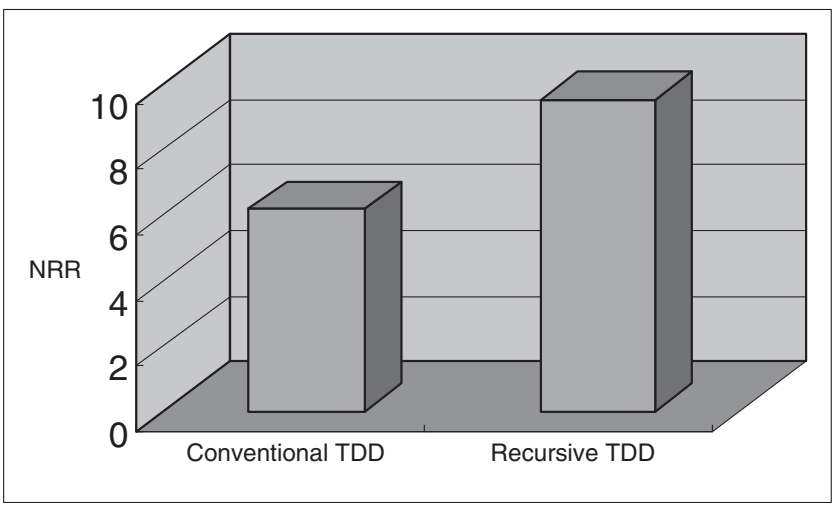

Fig. 8 Performance of recursive method against conventional TDD. The NRR $(\mathrm{dB})$ is averaged by 24 times of trials on different pairs of speeches.
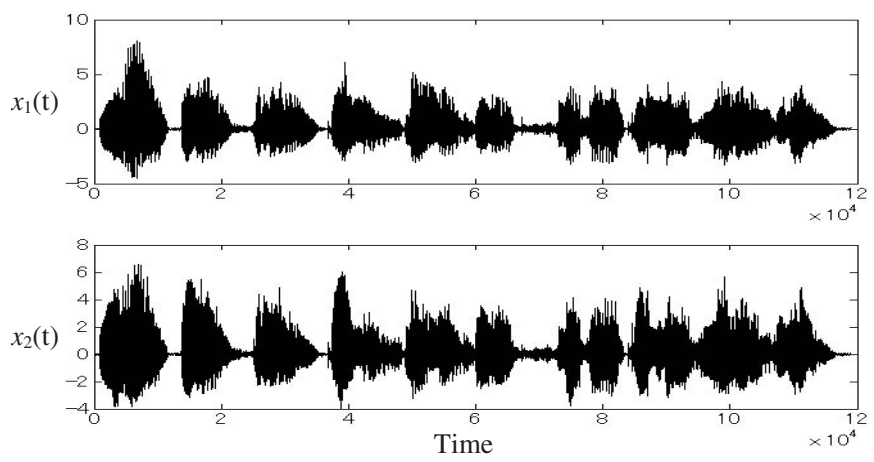

Fig. 9 Recorded signals in a real room.

\subsection{Test on Real Room Data}

The proposed algorithm was applied to the speech mixtures recorded in a real room. The data was downloaded from Prof. T. W. Lee's homepage (www.cnl.salk.edu/\%7Etewon). One speaker says the digits from one to ten in English and another speaker counts at the same time the digits in Spanish. The recording was done in a normal office room. Same parameters were used as those used in test 2 except for the threshold were set to 0.6 and 0.45 . The signals were sampled at $16 \mathrm{kHz}$, and about 2 seconds of signals were used to learn the filter. The waveforms of the two mixtures and the separated results are shown in Figs. 10 and 11, respectively. We evaluated the results by auditory inspection and confirmed that they were quite clear and better than those achieved by conventional method. The results are available at our home page (http://www.tuat.ac.jp/ khbase/members/hu/).

\section{DISCUSSION AND CONCLUSION}

We proposed a recursive algorithm for lowering the unfavorable effect from the collapse of independence occurred in frequency domain implementation. In this paper, we combined it with the TDD based blind separation
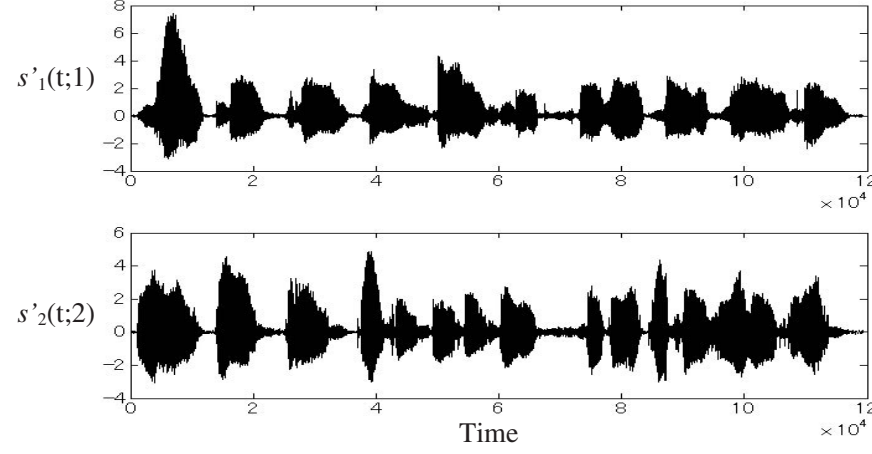

Fig. 10 The separated signals.

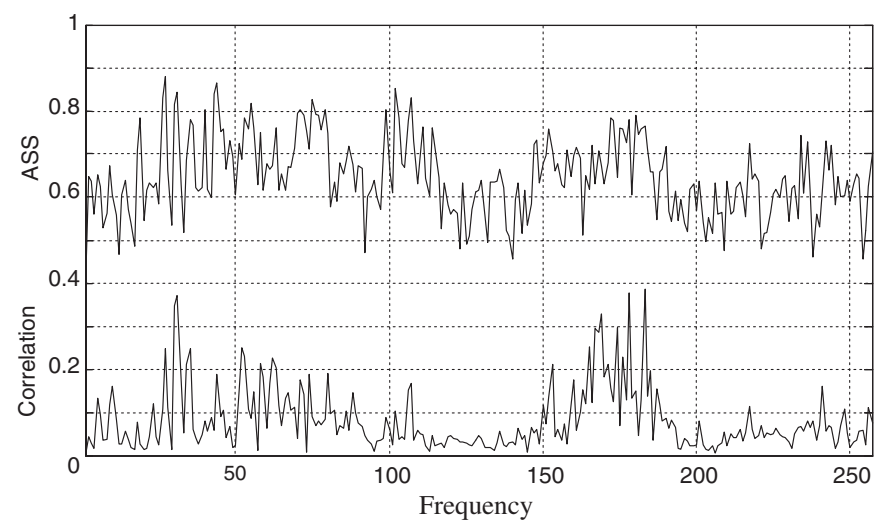

Fig. 11 The upper drawing is the ASS distribution after the conventional TDD has been implemented. The lower drawing is the correlation distribution. The two distributions are concerning the same sources.

method proposed by S. Ikeda and N. Murata. Simulation tests demonstrated its effectiveness.

Normally, it is very difficult to make the judgment whether a separation of mixed bin is good or not. We proposed using the ASS of the separated frequency components for this purpose. Figure 11 shows an example of ASS distribution against the correlation distribution between the original sources. The distribution of ASS is basically in consistence with that of correlation. Nevertheless, there exists some mismatching between them, which results in a few of improper separations yet to be further handled, and a few of acceptable separations were judged improper. Blind separation is still a very complicated problem. To which degree the partial correlation affects the separation is not clear. In some situation, the partial correlation and ASS are quite weak, but good separation has not been implemented.

In this paper, we only combined our method with time structure based decorrelation method. Theoretically, it is possible to combine method with any other blind separation methods for which the independence is assumed, and better separation can be expected. 


\section{ACKNOWLEDGEMENTS}

This work is supported in part by the Grant-in-Aid for Scientific Research from the Ministry of Education, Culture, Sports, Science and Technology, Japan.

\section{REFERENCES}

[1] S. Ikeda and N. Murata, "A method of blind separation based on temporal structure of signals," Proc. 5th Int. Conf. on Neural Information Processing (ICONIP '98 Kitakyushu), pp. 737-742 (1998).

[2] L. Tong, R.-W. Liu, V. C. Soon and Y.-F. Huang, "Indeterminacy and identifiability of blind identification," IEEE Trans. Circuits Syst., 38, 499-509 (1991).

[3] L. Molgedey and H. G. Schuster, "Separation of a mixture of independent signals using time delayed correlations," Phys. Rev. Lett., 72, 3634-3637 (1994).

[4] A. Belouchrani, K. Abed-Meraim, J.-F. Cardoso and E. Moulines, "A blind source separation technique using secondorder statistics," IEEE Trans. Signal Process., 45, 434-443 (1997).

[5] P. Comon, "Independent component analysis, a new concept?” IEEE Trans. Signal Process., 36, 287-314 (1994).

[6] A. Cichocki R. Unbehauen and E. Runmmert, "Robust learning algorithm for blind separation of signals," IEEE Electron. Lett., 43, 1386-1387 (1994).

[7] M. Girolami, "An alternative perspective on adaptive independent component analysis algorithms," Neural Comput., 10, 2103-2114 (1998).

[8] A. Hyvarinen and E. Oja, "A fast fixed-point algorithm for independent component analysis," Neural Comput., 9, 14831492 (1997).

[9] T.-W. Lee, A. Bell and T. Sejnowski, "Independent component analysis using an extended infomax algorithm for mixed sub-Gaussian and super-Gaussian sources," Neural Comput., 11, 609-633 (1999).

[10] A. Bell and T. Sejnowski, "An information maximization approach to blind separation and blind deconvolution," Neural Comput., 7, 1129-1159 (1995).

[11] S. Amari, A. Cichocki and H. H. Yang, "A new learning algorithm for blind signal separation," in Advances in Neural Information Processing Systems, Vol. 8, D. S. Touretzky, M. C. Mozer and M. E. Hasselmo, Eds. (MIT Press, Cambridge, MA, 1996), pp. 757-763.

[12] J. F. Cardoso and B. H. Laheld, "Equivalent adaptive source separation," IEEE Trans. Signal Process., 44, 3017-3030 (1996).

[13] K. Torkkola, "Blind separation for audio signals - Are we there yet?" Proc. Workshop on Independent Analysis and Blind Signal Separation, Jan. 11-16, France (1999).
[14] K. Torkkola, "Blind separation of convolved sources based on information maximization," IEEE Workshop on Neural Networks for Signal Processing, pp. 423-432 (1996).

[15] P. Smaragdis, "Blind separation of convolved mixtures in the frequency domain," Neurocomputing, 22, 21-34 (1998).

[16] F. Asano, Y. Motomura, H. Asoh and T. Matsui, "Effect of PCA filter in blind source separation," Proc. ICA 2000, pp. 57-62 (2000).

[17] S. Kurita, H. Saruwatari, S. Kajita, K. Takeda and F. Itakura, "Evaluation of blind signal separation using directivity pattern under reverberant conditions," Proc. ICASSP 2000, Vol. V, pp. 3140-3143 (2000).

[18] T.-W. Lee, A. Ziehe, R. Orglmeister and T. J. Sejnowski, "Combining time-delayed decorrelation and ICA: Towards solving the cocktail party problem," Proc. ICASSP 98, Vol. 2, pp. 1249-1252 (1998).

[19] T. Nishikawa, S. Araki and S. Makino, "Optimization on the number of subbands in blind source separation with subband ICA," Proc. Spring Meet. Acoust. Soc. Jpn., pp. 569-570 (2001).

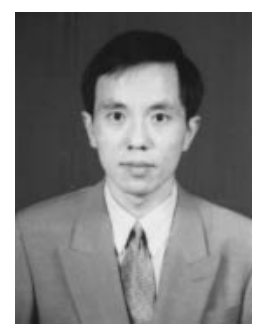

Xuebin Hu was born in Shanghai, China in April 1966. He received the B.S. degree in Engineering Physics from Tsinghua University in 1989 and the M.E. degree in Bio-applications and systems engineering from Tokyo University of Agriculture and Technology in 2001 respectively. He worked at the Dongfang Electric Co., China from 1989 to 1998 . He is currently a Ph.D. student at Graduate School of BioApplications and Systems Engineering, Tokyo University of Agriculture and Technology, Japan.

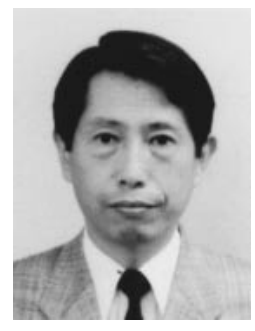

Hidefumi Kobatake received the B.E., M.E., and Ph.D. degrees from the University of Tokyo, Tokyo, Japan, in 1967, 1969 and 1972, respectively. From 1972 to 1975 he was an Research Associate at the Institute of Space and Aeronautical Science, University of Tokyo, Tokyo, Japan. Since 1975 he has joined the Tokyo University of Agriculture and Technology, Tokyo, Japan, where he is now a Professor at the Graduate School of Bio-Applications and Systems Engineering. During 1983-1984 he was an visiting Associate Professor at the School of Electrical Engineering, Georgia Institute of Technology, Atlanta. His research activities include areas of speech processing, image processing, and the applications of digital signal processing. $\mathrm{He}$ is the author of books on speech recognition, digital signal processing, mathematical morphology etc. 